\title{
高效率单光子量子存储
}

\author{
李传锋
}

中国科学技术大学, 中国科学院量子信息重点实验室, 合肥 230026

E-mail: cfli@ustc.edu.cn

\section{Efficient quantum memory for single photon}

\section{Chuanfeng Li}

CAS Key Laboratory of Quantum Information, University of Science and Technology of China, Hefei 230026, China E-mail: cfli@ustc.edu.cn

doi: 10.1360/TB-2019-0485

量子存储是量子信息领域的关键技术之一, 在量子 通信和量子计算等方向均有重要应用 ${ }^{[1]}$. 比如, 量子中继 是实现长程量子通信的重要方案, 而量子存储器则是构成 量子中继的基本元件之一. 经典信息的基本单元是比特, 只有 0 和 1 两种状态, 而量子信息的基本单元是量子比特, 可以处于量子叠加态. 光子具有传输速度快、与环境耦合 弱等优点, 是量子信息的天然载体. 量子存储就是把光子 所携带的量子态相干地转换到存储介质中, 并尽量使光子 不被损耗掉; 存储一段时间后, 等需要用这个量子态时还 能把它相干地转换回光子上. 衡量量子存储过程品质的指 标有存储保真度、存储效率、存储时间等 ${ }^{[2]}$. 要想实现量 子中继等功能, 需要提升量子存储的各项指标. 常见的量 子存储介质有冷原子 ${ }^{[3]}$ 、热原子 ${ }^{[4]}$ 、腔内单个原子 ${ }^{[5]}$ 和稀土 离子掺杂晶体 ${ }^{[6]}$ 等系统. 每种量子存储介质都有自己的特 点, 在提升各项指标的过程中也会遇到各自的问题.

冷原子系统具有与光的相互作用强等优点, 弱相干光
证实其可以被用来实现高效率的量子存储. 然而要实现单 光子的高效率量子存储需要解决两个难题: (1) 抑制噪声. 由于信号是单光子, 所以必须把噪声抑制到远小于单个光 子的水平. (2) 单光子和冷原子系统的模式匹配. 只有二者 模式匹配, 才能把单光子的量子态转换成冷原子的量子态 进行存储. 华南师范大学朱诗亮与颜辉课题组和香港科技 大学杜胜望教授等人 ${ }^{[7]}$ 合作在Nature Photonics 上发表题为 "Efficient quantum memory for single-photon polarization qubits”的研究论文. 他们通过长期的理论探索和实验设计, 解决了冷原子系统中的以上两个难题. 实验运用二维磁光 阱技术, 将钕原子温度冷却到 $200 \mu \mathrm{K}$, 并获得长条形冷原 子团; 结合暗线磁光阱和塞曼子能级制备技术, 将冷原子 的光学厚度提高到了 500 . 通过把控制光调整到和信号光 不重合, 而是有个 $2.2^{\circ}$ 的夹角, 有效解决了抑制噪声难题. 单光子源由另一个磁光阴中的铭原子团通过触发产生, 利 用空间光调制技术有效解决了单光子和冷原子系统模式匹
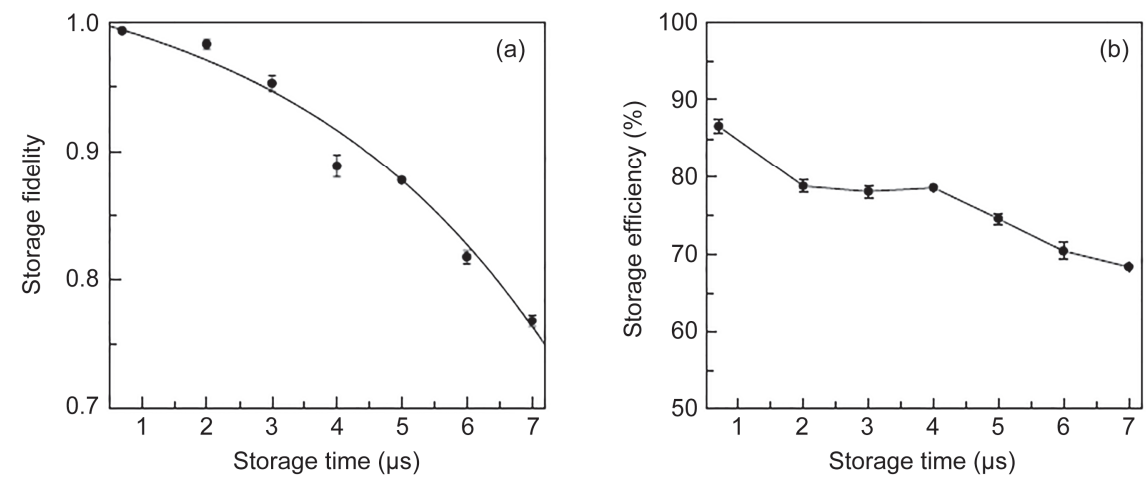

图 1 量子存储的保真度 $(a)$ 与存储效率 $(b)^{[7]}$

Figure 1 Storage fidelity (a) and storage efficiency (b) of quantum memory ${ }^{[7]}$ 
配难题. 最后利用电磁诱导透明技术实现了单光子存储效 率 $90.6 \%$. 通过对单光子偏振编码进行存储实验, 得到了高 达 $85 \%$ 的量子存储效率和 $99 \%$ 的保真度(图1).

该实验中的量子存储效率创造了目前世界最高水平,
是量子存储领域的重要进展之一. 本存储过程尚需提高之 处在于存储时间只有微秒量级, 而要想在量子中继等方向 有实际应用, 存储时间需要达到毫秒量级, 可行的方法有 进一步提高真空度和增大束缚势等.

\section{参考文献}

1 Duan L M, Monroe C. Colloquium: Quantum networks with trapped ions. Rev Mod Phys, 2010, 82: 1209-1224

2 Simon C, Afzelius M, Appel J, et al. Quantum memory. Eur Phys J D, 2010, 58: 1-22

3 Matsukevich D N, Kuzmich A. Quantum state transfer between matter and light. Science, 2004, 306: 663-666

4 Hosseini M, Campbell G, Sparkes B M, et al. Unconditional room-temperature quantum memory. Nat Phys, 2011, 7: 794-798

5 Specht H P, Nölleke C, Reiserer A, et al. A single-atom quantum memory. Nature, 2011, 473: 190-193

6 de Riedmatten H, Afzelius M, Staudt M U, et al. A solid-state light-matter interface at the single-photon level. Nature, 2008, 456: 773-777

7 Wang Y F, Li J F, Zhang S C, et al. Efficient quantum memory for single-photon polarization qubits. Nat Photonics, 2019, 13: 346-351 